\title{
Apoyo familiar" el éxito del uso del audífonos en el paciente con hipoacusia en el Hospital Pablo Arturo Suarez Quito - Ecuador.
}

Family support" the success of the use of hearing aids in the patient with hearing loss in the Hospital Pablo Arturo Suárez Quito - Ecuador.

Msc. Salome Ruales. ${ }^{1}$, Dr. Fausto Martinez. ${ }^{2}$, Lcda. Inés Chávez. ${ }^{3} \&$ Dr. Diego Noboa. ${ }^{4}$

Recibido: 05-03-2017 / Revisado: 08-05-2017 Aceptado: 07-06-2018/ Publicado: 01-07-2018

\begin{abstract}
.
DOI: https://doi.org/10.33262/cienciadigital.v2i3.164

Hearing disability is a bio psychosocial problem that is associated with difficulties in the development of daily activities and interpersonal relationships, which affect the quality of life of people who suffer this disability.

The present investigation aims to know how the use of hearing aids contributes greatly to improving the living conditions of people with this type of disability and the positive effect and importance of the environment in the independence and inclusion in society of these people.

The accompaniment of the relatives in the process of delivery, use, maintenance of the equipment, acceptance and inclusion in a social context, has been fundamental to obtain the desired results in this research.

149 patients were given hearing aids, for this study 101 users were followed up for six months.

An audiological evaluation (audiometry, speech audiometry, supra-liminal tests) was performed for this investigation. The day of delivery trained has given about handling and use of the hearing aid to patients and their families.
\end{abstract}

\footnotetext{
${ }^{1}$ Universidad Central de Ecuador, Facultad de Ciencias de la discapacidad, Quito, Ecuador, salomerualesparedes@gmail.com

2 Jefe del Servicio de Otorrinolaringología, Quito, Ecuador, fagusmartinezent@gmail.com

${ }^{3}$ Hospital Pablo Arturo Suarez, Terapista del Lenguaje, Quito, Ecuador, inlu27@hotmail.com

${ }^{4}$ Hospital Pablo Arturo Suarez, Gestión de Docencia e Investigación, Quito, Ecuador, diego.noboa@hpas.gob.ec
} 
Interesting data were obtained on the improvement in the quality of life of patients with hearing loss, this relevant information helped to establish how the living conditions of this population can be better.

Keywords: Hearing aid, Quality of Life, Hearing Loss, Family Support.

\section{Resumen.}

La discapacidad auditiva es un problema biopsicosocial que está asociado con dificultades en el desenvolvimiento de actividades cotidianas y relaciones interpersonales, que afectan la calidad de vida de las personas que lo padecen.

La presente investigación está encaminada en conocer, cómo el uso de Audífonos, aporta en gran medida a mejorar las condiciones de vida de las personas con este tipo de discapacidad y el efecto positivo e importancia del entorno en la independencia e inclusión en la sociedad de estas personas.

El acompañamiento de los familiares en el proceso de entrega, uso, mantenimiento de los equipos, aceptación e inclusión en un contexto social, ha sido fundamental para obtener los resultados deseados en esta investigación.

Se entregaron audífonos a 149 pacientes, para este estudio y se tomaron como muestra 101 usuarios del dispositivo durante seis meses.

Para esta investigación se realizó una evaluación audiológica (audiometría, logo audiometría, pruebas supra liminares), talleres de sensibilización a los familiares, y el día de la entrega se capacitó en el manejo y uso del audífono a pacientes y familiares.

Se obtuvieron datos interesantes sobre la mejora en la calidad de vida de los pacientes con hipoacusia, esta información relevante ayudo a establecer cómo se pueden mejor las condiciones de vida de esta población.

Palabras Claves: Audífono, Calidad de Vida, Hipoacusia, Apoyo Familiar.

\section{Introducción .}

La hipoacusia es una condición muy frecuente principalmente en las personas adultas mayores, según la Organización Panamericana de la Salud más del 5\% de la población mundial (360 millones de personas) padece pérdida de audición incapacitante (328 millones de adultos y 32 millones de niños). Esta se entiende a una pérdida de audición superior a 40 $\mathrm{dB}$ en el oído con mejor audición en los adultos, y superior a $30 \mathrm{~dB}$ en el oído con mejor audición en los niños. Fuente: [1] 1 de Julio del 2017. 
Aproximadamente una tercera parte de las personas mayores de 65 años padece pérdida de audición, en ECUADOR según datos estadísticos del CONADIS el porcentaje de discapacidad en el país es de $12.81 \%$, siendo la tercera causa de discapacidad en el País, Fuente: [2] 28 de junio del 2107. La Discapacidad Auditiva trae consigo problemas psicológicos como aislamiento, ansiedad, depresión, dependencia, que reducen la calidad de vida de estas personas.

Afortunadamente en la actualidad la tecnología ha permitido que se desarrollen dispositivos que permiten a la persona con discapacidad auditiva volver a escuchar y de esta manera retomar su independencia y autonomía en las actividades de la vida diaria.

\section{Objetivo general.}

Determinar la influencia del uso de audífono retro auricular en personas con hipoacusia y su desempeño en la sociedad.

\section{Objetivos especificos:}

- Involucrar y motivar a los familiares en el proceso de adaptación social.

- Fomentar la enseñanza para el buen manejo y cuidado diario de los audífonos.

\section{Materiales y métodos.}

El diseño de la investigación es de tipo descriptivo prospectivo, en un periodo de seis meses para analizar cómo ha mejorado la calidad de vida en personas con discapacidad auditiva una vez que son usuarios de audífonos.

En el Hospital Pablo Arturo Suárez al año se evalúan aproximadamente 2500 usuarios de los cuales se han registrado hasta el presente estudio 538 pacientes que requieren audífonos, para la selección de la población se tomaron en cuenta parámetros de inclusión y exclusión según los lineamientos enviados por el Ministerio de Salud Pública.

Se evidenció que la población beneficiada inicialmente fue de 149 pacientes los que recibieron su audífono y son parte de este estudio.

Dentro de la evaluación se realizaron dos tipos de encuestas. Una dirigida a los pacientes, encaminada a conocer sobre el uso y manejo del audífono, colocación de baterías, tiempo de uso diario, actividades en las que lo utilizan y si su vida diaria se ha modificado con el mismo.

En relación a la encuesta dirigida a la familia se abordaron temas relacionados a la percepción de cambios favorables en el comportamiento y desenvolvimiento en actividades de la vida diaria de las personas con discapacidad auditiva. Se -involucró a la familia en talleres vivenciales para sensibilizarlos sobre la problemática en la que viven las personas con sordera y cómo afrontarla. 
Estas encuestas fueron realizadas en dos momentos: el primero antes de la adaptación de audífonos y la segunda seis meses luego de la adaptación de audífonos.

Esta información fue procesada y tabulada utilizando el programa Excel.

\section{Resultados.}

La primera encuesta realizada a las personas con hipoacusia antes de haber sido adaptadas con audífonos retroauriculares, demostró que la mayoría tenía dificultad para comunicarse y relacionarse con sus familiares, amigos y conocidos provocando que se aíslen, desembocando en ocasiones en problemas emocionales como ansiedad y depresión, esto por falta de comprensión por parte de sus familiares y de la sociedad en general, de igual manera el desempeño en la realización de actividades de la vida diaria se ven disminuidas afectando la independencia y generando mucha inseguridad, todos estos aspectos dan como resultado un descenso en la calidad de vida.

La segunda evaluación con la misma encuesta, se realizó después de 6 meses de haberse entregado los audífonos retro auriculares a un total de 101 pacientes. En base a la información recopilada se evidencia que la mayoría de las personas con discapacidad auditiva han mejorado su calidad de vida desde el uso del dispositivo, un gran porcentaje de los usuarios mencionan que el audífono les ha ayudado en la realización de sus actividades diarias obteniendo independencia, seguridad y elevando su autoestima. La mayoría de pacientes y sus familiares comentan que a partir del uso de las prótesis auditivas se comunican de mejor manera, interviniendo en las conversaciones e involucrándose más en reuniones familiares, así mismo los talleres realizados para concientizar a los familiares sobre la discapacidad auditiva dieron muy buenos resultados logrando que las personas entiendan la problemática de las personas con discapacidad auditiva y de esta manera brinden el apoyo necesario en el proceso de adaptación.

Tabla 1. Mejora en la calidad de vida

\begin{tabular}{lll}
\hline SI & 85 & $84 \%$ \\
\hline NO & 16 & $16 \%$ \\
TOTAL & 101 & $100 \%$ \\
\hline
\end{tabular}

Fuente: Audiología 2017 
Gráfica 1. Mejora en la calidad de vida.

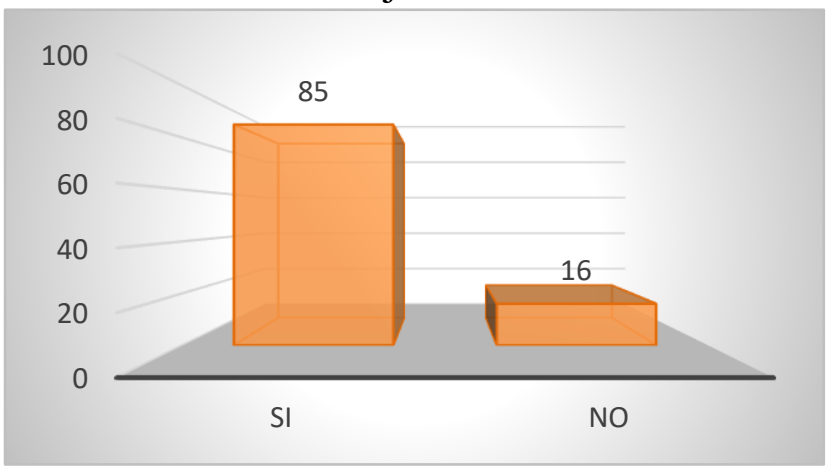

Fuente: Audiología 2017.Figura 1. ¿Cree usted que ha mejorado sus condiciones de vida con el uso del audífono?

El $84 \%$ de las personas adaptadas creen que el uso del audífono ha mejorado sus condiciones de vida y el $16 \%$ indica que no ha tenido ningún cambio.

Tabla 2. Independencia en la realización de actividades de la vida diaria

\begin{tabular}{lcc}
\hline SI & 88 & $87 \%$ \\
\hline NO & 13 & $13 \%$ \\
TOTAL & 101 & $100 \%$ \\
\hline
\end{tabular}

Fuente: Audiología 2017.

Gráfica 2. Independencia en la realización de actividades de la vida diaria

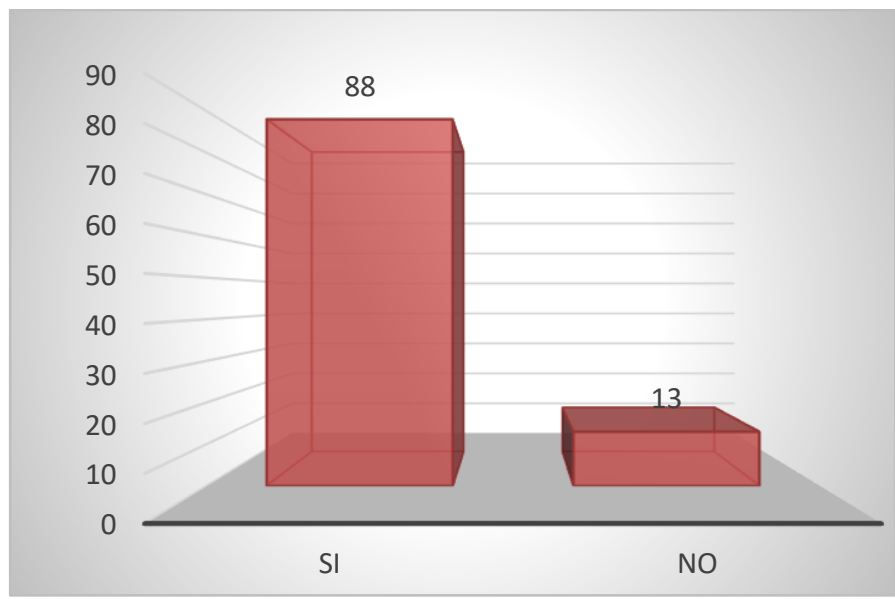

Fuente: Audiología 2017. 
Figura 2. El audífono le ha ayudado en actividades diarias

Con respecto a las actividades de la vida diaria el $87 \%$ de las personas mencionan que el audífono les ha ayudado en sus actividades diarias y al $13 \%$ no.

Tabla 3. Mejoría en la comunicación con las personas

\begin{tabular}{|l|l|l|}
\hline SI & 74 & $73 \%$ \\
\hline NO & 18 & $18 \%$ \\
\hline A VECES & 9 & $9 \%$ \\
\hline TOTAL & 101 & $100 \%$ \\
\hline
\end{tabular}

Fuente: Audiología 2017

Gráfica 3. Mejoría en la comunicación con las personas

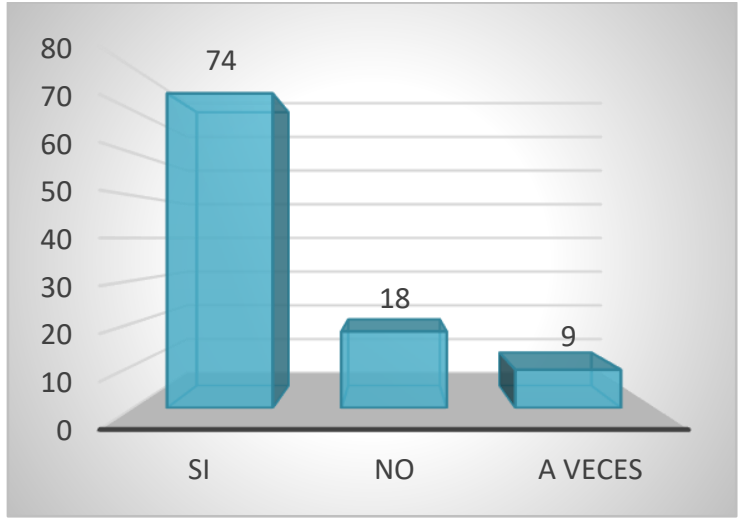

Fuente: Audiología 2017

Figura 3. ¿Sus familiares, amigos y/o conocidos refieren que se comunica de mejor manera desde que usa el audífono?

Al 73\% de las personas sus familiares y/o conocidos les han dicho que se comunican mejor desde la adaptación de los audífonos, al $18 \%$ no le han dicho nada y al $9 \%$ a veces les han mencionado el tema. 
Tabla 4. Progreso en las relaciones con familiares.

\begin{tabular}{|l|l|l|}
\hline SI & 94 & $93 \%$ \\
\hline NO & 7 & $7 \%$ \\
\hline TOTAL & 101 & $100 \%$ \\
\hline
\end{tabular}

Fuente: Audiología 2017

Tabla 4. Progreso en las relaciones con familiares.

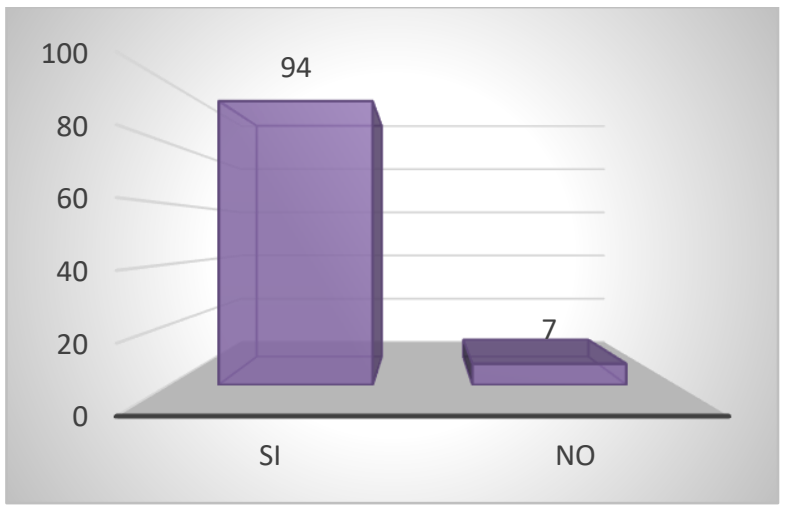

Fuente: Audiología 2017.

Figura 4. ¿Cree usted que los talleres le permitieron mejorar las relaciones con sus familiares amigos y conocidos?

Se observa que el $93 \%$ de las personas adaptadas con prótesis auditivas cree que los talleres les ha permitido mejorar sus relaciones familiares y el $7 \%$ indica que no ha mejorado.

En los resultados obtenidos refirieron los beneficiados de audífonos mayor independencia en un $83 \%$, el 17\% indica que no tienen mayor independencia. El $73 \%$ de los familiares y/o conocidos encuestados refieren que perciben una mejoría en su familiar. Con respecto a las actividades de la vida diaria el $87 \%$ de las personas mencionan que el audífono les ha ayudado en sus actividades diarias y al 13\% no. El $84 \%$ de las personas adaptadas creen que el uso del audífono les ha cambiado la vida y el $16 \%$ indica que no ha tenido ningún cambio

\section{Discusion.}

La discapacidad auditiva es un problema médico y social que afecta el desenvolvimiento de la persona en la vida cotidiana, por este motivo, el usar audífonos le permite insertarse nuevamente en la sociedad puesto que ya no se aísla del entorno donde se desenvuelve.. Uno de los factores importantes para asegurar el uso y adaptación del audífono, fueron los 
talleres teórico-práctico donde se enseñó el manejo y uso del audífono. Así como sensibilizar y capacitar a la familia.

Los factores que influyen en el uso del audífono que le permitió mejorar la calidad de vida en las personas con pérdida auditiva es la dificultad de manejar los audífonos, así como la colocación del mismo, esta situación se presenta principalmente en las personas adultas mayores o aquellas que no tienen apoyo familiar.

Otro punto que influyo es la compresión de los familiares para apoyar este proceso y comprender que el audífono facilita la escucha, aclarando que sus respuestas auditivas no van hacer lo mismo que cuando tenía audición normal. Se evidencio que los pacientes mejor adaptados son aquellos a quien la familia les tiene mayor paciencia y se toman el tiempo necesario para llevarle a los controles.

Se observa que el 93\% de las personas adaptadas con prótesis auditivas cree que el taller les ha permitido mejorar sus relaciones familiares y el $7 \%$ indica que no ha mejorado, esto se debe a que la persona que acudió a los talleres por tiempo, distancia, no puede estar siempre cerca del paciente, debido a que no vive con él o ella o reside en otro sector de la ciudad, otro factor es que a los talleres acudieron personas con dificultad en adquirir nuevos aprendizajes.

En el país no se evidencian estudios similares sobre la mejora en la calidad de vida que genera el uso de audífonos, por lo que esta investigación inicia las bases para próximas investigaciones.

El trabajo realizado ha sido enriquecedor en base a la intervención como profesional y a la retroalimentación de los familiares y pacientes a quienes está dedicada la presente.

\section{Conclusiones.}

- Del seguimiento realizado a los pacientes con hipoacusia que fueron beneficiarios de los audífonos retro auriculares, se determinó una influencia directa y positiva en el desenvolvimiento de actividades diarias de manera independiente de las personas con hipoacusia, que esto a su vez llevo a una mejora en su autoestima, así como una motivación para socializar y mejorar las habilidades comunicativas e interpersonales.

- Se observa además que una de las actividades que generó impacto fueron los talleres realizados tanto al paciente como a sus familiares, sobre el manejo y uso correcto de los equipos, esto produjo una mayor interacción con los pacientes creando empatía y consideración con sus familiares logrando que apoyen activamente en este proceso.

- En las personas que indican que el uso del audífono no ha mejorado su calidad de vida, puede estar relacionado a una alteración en la discriminación o comprensión del lenguaje relacionado al tiempo que no ha escuchado. Un factor dominante para 
crear nuevos hábitos de uso de los audiófonos retro auriculares, fue el apoyo familiar, quienes motivaron a los pacientes a continuar con el uso de los audífonos incluso al haberse presentado ciertas molestias e incomodidades.

- Una de las dificultades presentadas fueron las expectativas altas que tienen los beneficiarios al pensar que escuchara con audición normal, siendo la utilización de estos equipos un apoyo en la mejora de audición y por ende todo lo que conlleva el escuchar.

\section{Referencias bibliográficas.}

Organización Mundial de la Salud. (Febrero 2017). Sordera y pérdida de la audición. Recuperado el 25 de junio de 2017 de: http://www.who.int/mediacentre/factsheets/fs300/es/

Conadis http://www.consejodiscapacidades.gob.ec/estadistica/index.html

Pedraza, z. Delgado, m. (mayo-junio 2008). El déficit de audición en la tercera edad. Medigraphic Artemisa en línea. Recuperado el 24 de junio de 2017 de: http://www.medigraphic.com/pdfs/facmed/un-2008/un083b.pdf

Moreno, Raquel. (2015 - 2016). PLASTICIDAD CEREBRAL Y DETERIORO COGNITIVO EN ANCIANOS CON AUDÍFONOS. (Técnico superior en audiología protésica). E. U. GIMBERNAT CANTABRIA. Torrelavega. Recuperado el 24 de junio de 2017 de: http://eugdspace.eug.es/xmlui/bitstream/handle/123456789/213/Raquel\%20Moreno\% 20Soler.pdf? sequence $=1$

Farfán, c. Aguilera, e. Lecaros, r. Riquelme, k. Valenzuela, m. Manque, p. (2015). NO ADHERENCIA AL USO DE AUDÍFONOS EN ADULTOS MAYORES DE 65 AÑOS. PROGRAMA GES, HOSPITAL CARLOS VAN BUREN, 2014. Rev Chil Salud Pública. Recuperado el 24 de junio de 2017de: http://www.auroradechile.uchile.cl/index.php/RCSP/article/viewFile/37636/39302

Pintado, Lilian. (Mayo 2016). Pérdida auditiva y habilidades cognoscitivas en adultos mayores. (Maestría en Ciencias en Patología del Habla-Lenguaje). Universidad del Turabo. Recuperado el 24 de junio de 2017 de: http://ut.suagm.edu/sites/default/files/uploads/Health-Sciences/Thesis/2016/KristyAlamo-Nathalie-Alvarez-Karen-Batista-PHL-2016.pdf

Tamblay, n. Villalobos, i. Pastene, a. Rahal, m. (2008). Impacto social del uso de audífonos en adultos mayores. Rev. Otorrinolaringol. Cir. Cabeza Cuello. Recuperado el 24 de junio de 2017 de: http://www.scielo.cl/scielo.php?pid=S0718$48162008000100004 \&$ script=sci_arttext\&tlng=pt 
Algaba, J. Altuna, X. (2013). Rasgos clínicos e impacto social de la presbiacusia. LIBRO BLANCO SOBRE LA PRESBIACUSIA. (pp. 42 - 45). Disponible en http://www.lacomunidadpublicitaria.com/files/field/file/libro_presbiacusia_ok.pdf\#pa ge $=29$

Arteaga, Daniela. Correa, Sebastián. Duque, Karen. Ruiz, Sara. (2013). ABORDAJE PREHOSPITALARIO DEL PACIENTE CON DISCPACIDAD AUDITIVA. UNIVERSIDAD CES FACULTAD DE MEDICINA TECNOLOGIA EN ATENCIÓN PREHOSPITALARIA MEDELLÍN. Recuperado el 24 de junio de 2017 de:

http://bdigital.ces.edu.co:8080/repositorio/bitstream/10946/2297/2/ABORDAJE_PRE HOSPITALARIO_PACIENTE_DISCPACIDAD_AUDITIVA.pdf

Andrade, Judit. (2015). Presbiacusia. Adherencia al uso de audífonos en personas mayores. (GRADO/MÁSTER EN: GERONTOLOGÍA). UNIVERSIDADE DA CORUÑA. Recuperado el 25 de junio de 2017 de: http://ruc.udc.es/dspace/handle/2183/14515

Cardemil, f. Muñoz, d. Fuentes, e. (2016). Hipoacusia asociada al envejecimiento en Chile: ¿En qué aspectos se podría avanzar? Rev. Otorrinolaringol. Cir. Cabeza Cuello. Recuperado el 25 de junio de 2017 de: http://www.scielo.cl/scielo.php?pid=S0718$48162016000100018 \&$ script=sci_arttext\&tlng=pt

Cambala, Mónica. (2015-2016). ESTRATEGIA ALTERNATIVA DE INCLUSIÓN SOCIAL PARA EL MEJORAMIENTO DE LAS DE VIDA DE LAS PERSONAS CON DISCAPACIDAD AUDITIVA EN EL CANTÓN LA LIBERTAD, PROVINCIA DE SANTA ELENA, PERÍODO 2014-2016" (LICENCIADA EN DESARROLLO COMUNITARIO). UNIVERSIDAD ESTATAL PENÍNSULA DE SANTA ELENA. Recuperado el 25 de junio de 2017 de: http://repositorio.upse.edu.ec/bitstream/46000/1924/1/UPSE-TOD-2015-0020.pdf

Díaz, c. Goycoolea, m. Cardemil, f. (2016). HIPOACUSIA: TRASCENDENCIA, INCIDENCIA Y PREVALENCIA. Revista Médica Clínica Las Condes. Recuperado el 25 de junio de 2017 de: http://www.sciencedirect.com/science/article/pii/S0716864016301055

Lasso, r. (2012). Intervención en Salud Pública. Promoción de la salud auditiva en mujeres gestantes. Una propuesta de intervención. Revista Electrónica de Portales Médicos. Recuperado el 25 de junio de 2017 de: http://www.portalesmedicos.com/publicaciones/articles/4833/1/Intervencion-enSalud-Publica.-Promoocion-de-la-salud-auditiva-en-mujeres-gestantes.-Unapropuesta-de-intervencion 
Hear-it. (Julio 2010). La pérdida de audición sin tratar puede causar problemas sociales y psicológicos. Recuperado el 25 de junio de 2017 de: http://www.hear-it.org/es/laperdida-de-audicion-sin-tratar-puede-causar-problemas-sociales-y-psicologicos

Hear-it. (Julio 2014). Múltiples consecuencias de la pérdida de audición. Recuperado el 25 de junio de 2017 de: http://www.hear-it.org/es/multiples-consecuencias-de-laperdida-de-audicion

Deleg, Ruth. Saca, Ana. (2016). “CARACTERÍSTICAS SOCIODEMOGRÁFICAS DEL ADULTO MAYOR CON DETERIORO AUDITIVO EN EL CENTRO DE ATENCIÓN DEL IESS. CUENCA 2015". (PROYECTO DE INVESTIGACIÓN PREVIO A LA OBTENCIÓN DE TÍTULO DE MÉDICA). UNIVERSIDAD DE CUENCA. Recuperado el 25 de junio de 2017 de: http://dspace.ucuenca.edu.ec/bitstream/123456789/25359/1/TESIS.pdf

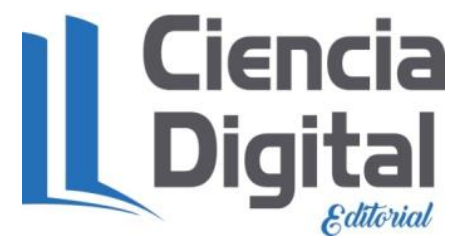


Para citar el artículo indexado.

Ruales S., Martínez F., Chavéz I. \& Noboa D. . (2018). “Apoyo familiar” El éxito del uso del audífonos en el paciente con hipoacusia en el Hospital Pablo Arturo Suarez Quito Ecuador. Revista electrónica Ciencia Digital 2(3), 382-393. Recuperado desde: http://cienciadigital.org/revistacienciadigital2/index.php/CienciaDigital/article/view/164/14 $\underline{4}$

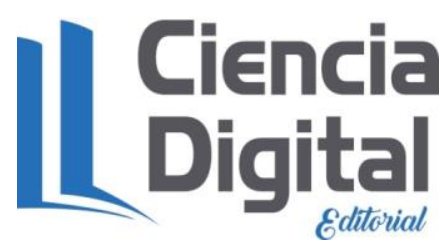

El artículo que se publica es de exclusiva responsabilidad de los autores y no necesariamente reflejan el pensamiento de la Revista Ciencia Digital.

El articulo queda en propiedad de la revista y, por tanto, su publicación parcial y/o total en otro medio tiene que ser autorizado por el director de la Revista Ciencia Digital.
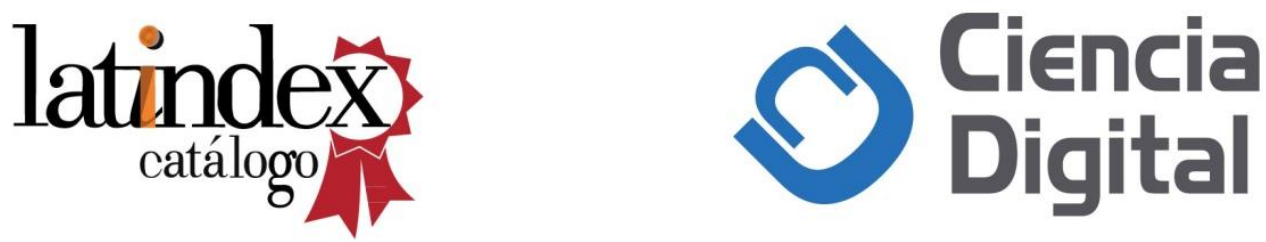